\title{
A New Type of Polymer Carbon Dots with High Quantum Yield: from Synthesis to Investigation on Fluorescence Mechanism
}

\author{
Songyuan Tao ${ }^{\mathrm{a}}$, Yubin Song ${ }^{\mathrm{a}}$, Shoujun Zhu ${ }^{\mathrm{a}, \mathrm{b}}$, Jieren Shao ${ }^{\mathrm{a}}$, Bai Yang ${ }^{\mathrm{a}, *}$ \\ ${ }^{\text {a }}$ State Key Lab of Supramolecular Structure and Materials, College of Chemistry, \\ Jilin University, Changchun 130012, P. R. China. \\ ${ }^{b}$ Department of Chemistry, Stanford University, Stanford, CA 94305, USA.
}

\begin{abstract}
Carbon dots (CDs) is an area of intense focus, currently limited to unclear photoluminescence (PL) mechanism. Much effort has been made to clarify the certain PL centers but is still fruitless. Herein, a new type of polymer carbon dots (PCDs) with absolute quantum yield as high as $44.18 \%$ was synthesized by hydrothermally crosslinking polyacrylic acid and ethylenediamine. Through carefully comparing and analysing, the PL centers were supposed to be $-\mathrm{N}=\mathrm{O}$ and $-\mathrm{C}=\mathrm{O}$ (mainly amide and/or its derived structures) rather than large-scale conjugated structure, which was similar to the previously reported sub-fluorophores. Besides, the function of polymer chains was considered to decrease the motion freedoms and provide a solvation effect by crosslinking and wrapping the fluorescence centers, which led to the improved quantum yield and red-shifted emission. The investigation on fluorescence mechanism can provide with a new understanding on crosslink enhanced emission (CEE) effect in non-conjugated fluorescence systems.
\end{abstract}

\section{Introduction}

Carbon dots (CDs) [1-10] has obtained continuous attention owing to its prominent characters and wide application prospects since firstly reported [11]. CDs always possesses nano-sized dot structure and fluorescence as their instinct properties. Compared with the conventional fluorescent dyes, the carbon-based fluorescence material owns various advantages, such as facile synthesis, bright emission, low toxicity and good biocompatibility $[12,13]$. In the last few years, researchers have prepared a wide variety of CDs by exploiting plenty of preparation methods and the original materials, resulting in large differences on the structure, property and fluorescence mechanism. Up to now, the field of CDs has developed greatly but is also facing large challenges, such as the lack of long-wavelength-emission systems [14-16] and confused fluorescence mechanism [17-19]. More and more researchers are looking forward to finding a systematic way to explain the complicated phenomenon and expand the application fields, which may cause further development in the field of CDs.

In addition, fluorescent polymer is another kind of functional materials, which can be applied to the chemical and biological sensing [20,21]. Introducing functional fluorophores onto the polymer chain [22] and polymerizing conjugated monomer [23,24] are two common ways to obtain fluorescent polymer. However, many conjugated polymers suffer from low aqueous solubility and high toxicity, which hinder the direct application of biological sensing. Recent researches found that some non-conjugated polymers were instinctively emissive. Among all, poly(amido amine)s were typical models [25-29], which didn't own common fluorophores but still highly emissive in solution. The hyperbranched structure was inferred as an important factor for the emission [30]. Furthermore, other special fluorescence mechanisms of linear non-conjugated fluorescent polymers were gradually uncovered. For example, Song et al reported linear polymers containing pyrrolidone rings could exhibit strong fluorescence [31]. The fluorophores were considered to be secondary amine oxide and the strong emission was attributed to fluorophores' aggregation-induced emission effect. Zhao et al synthesized emissive poly[(maleic anhydride)-alt-(vinyl acetate)] and proved that its emission originated from anhydride cluster interacting in a through-space manner [32].

As their cousin, a new type of fluorescent material polymer carbon dots (PCDs) [17,33-36] is becoming a hot topic, which inherts the advantages of both sides. Typically, PCDs is nano-sized particle possessing abundant polymer structures with low carbonization degree, prepared from the monomers or non-conjugated polymers by condensation, crosslinking, assembling, or slightly 
carbonization processes. There is a relationship among the mechanisms of PCDs, CDs and non-conjugated fluorescent polymers, which is waiting to be discovered. Our group also put forward our own opinion on the emission origin of PCDs, previously. Zhu et al investigated the fluorescence from sub-fluorophores in the non-conjugated polymer or PCDs and found that emission could be enhanced by chemical crosslinking or physical immobilization of polymer chains, which was named the crosslink-enhanced emission (CEE) effect [17,33,34]. Based on the background above, we would like to share our new comprehension on the fluorescence mechanism through investigating a new type of PCDs.

In this paper, we synthesized PCDs with high quantum yield via a one-pot hydrothermal treatment of polyacrylic acid (PAA) and ethylenediamine (EDA). The obtained PCDs was confirmed to be crosslinked nanoparticles with low degree of carbonization and remian characters of polymer. The fluorescence source was inferred as the oxidation product of amine during hydrothermal process. Moreover, the PAA chains played a vital role in forming crosslinked polymer structure and wrapping the fluorophores. The decreased motion freedom led to enhanced emission intensity and the new chemical environment led to red-shifted emission from UV region to visible region. The explanation of PCDs' fluorescence mechanism could further refine our CEE effect theory.

\section{Experimental}

\subsection{Materials}

PAA $\left(\mathrm{M}_{\mathrm{w}}=3500 \mathrm{~g} / \mathrm{mol}\right)$, EDA and quinine sulfate were purchased from Aladdin Chemical Co. Sulfuricacid $\left(\mathrm{H}_{2} \mathrm{SO}_{4}, 98 \%\right)$ and hydrochloric acid $(\mathrm{HCl}, 36 \sim 38 \%)$ were obtained from Beijing Chemical Reagent Co. Potassium persulfate was purchased from Tianjin Huadong Chemical Reagent Co. All of the chemicals were used without further purification.

\subsection{The synthesis of PCDs}

PAA (72 mg, $1 \mathrm{mmol}-\mathrm{COOH})$ was dissolved in deionized water $(10 \mathrm{~mL})$. After that, EDA (140 $\mu \mathrm{L})$ was added and mixed uniformly. Then the solution was transferred to a poly (tetrafluoroethylene) (Teflon)-lined autoclave $(25 \mathrm{~mL})$ and heated at different temperatures in oven. However, higher temperature didn't lead to obvious promotion of PL intensity but great damage to autoclave. In comprehensive consideration of product's fluorescence intensity and autoclave's heat-resistance temperature, the experiment condition of $200^{\circ} \mathrm{C}$ for $8 \mathrm{~h}$ was selected for further studies. After the reaction, the reactors were cooled down to room temperature naturally. The obtained transparent buff solution was dialyzed in dialysis bag (500-1000 D) against deionized water for three days, in order to remove the small molecules. Solid PCDs could be obtained from the solution via freeze-drying. (yield ca. $30 \%$ )

\subsection{The synthesis of EDA-H}

Ethylenediamine (EDA, $140 \mu \mathrm{L})$ was added to deionized water $(10 \mathrm{~mL})$. Then the solution was transferred to a poly (tetrafluoroethylene) (Teflon)-lined autoclave $(25 \mathrm{~mL})$ and heated at $200^{\circ} \mathrm{C}$ for $8 \mathrm{~h}$. After the reaction, the reactors were cooled down to room temperature naturally. The colorless and transparent solution was filtered by $0.22 \mu \mathrm{m}$ polyehersulfone membrane to remove large particles. The product was named as EDA-H. In addition, other EDA hydrothermal products were synthesized by adding potassium persulfate $\left(\mathrm{K}_{2} \mathrm{~S}_{2} \mathrm{O}_{8}, 5,25\right.$ or $\left.200 \mathrm{mg}\right)$ in solution before heating.

\subsection{Characterization}

High-resolution transmission electron microscopy (HRTEM) was recorded on a JEOL JEM-2100F by using ultra-thin carbon films as grids. IR spectra were taken on a Nicolet AVATAR 360 FT-IR spectrophotometer. Zeta potential and DLS were performed using a Zetasizer Nano-ZS (Malvern Instruments). NMR spectra were performed with a Bruker AVANCE NMR spectrometer (500 MHz) using $\mathrm{D}_{2} \mathrm{O}$ as the solvent. Thermogravimetric analysis (TGA) was measured on a Mettler Toledo TGA/SDTA851e instrument under $\mathrm{N}_{2}$ atmosphere from room temperature to $600^{\circ} \mathrm{C}$ with a heating rate of $10^{\circ} \mathrm{C}$ per minute. X-ray photoelectron spectroscopy (XPS) was performed with an ESCALAB 250 spectrometer with a mono X-ray source with $\mathrm{Al} \mathrm{K} \alpha$ excitation $(1486.6 \mathrm{eV})$. UV-vis absorption spectra 
were obtained using a 3100 UV-vis spectrophotometer (Shimadzu). Static fluorescence spectra were performed on a RF-5301 PC spectrophotometer (Shimadzu). Fluorescence lifetime (with TCPSC technology) and emission spectra at different temperatures were collected on FLS 980 (Edinburgh). Absolute QY was measured on FLS 920 with an integrating sphere (Edinburgh). Excitation-emission matrix was recorded on a Fluoromax-4 spectrofluorometer (Horiba Jobin-Yvon).

\section{Results and discussion}

\subsection{A new kind of PCDs with high quantum yield}

A facile method was adopted to prepare PCDs with high quantum yield, excellent water solubility and low toxicity, by hydrothermal reaction of PAA and EDA (Figure 1). The optimal reaction condition was confirmed as $200^{\circ} \mathrm{C}$ and $8 \mathrm{~h}$ (Table S1). By optimizing the ratio of PAA and EDA (Table S2), a new kind of PCDs was gotten with an absolute quantum yield as high as $44.18 \%$ in solution. According to the different characters and wavelengths of emission peaks in PCDs' PL spectra (Figure 2a), two major fluorescence centers could be distinguished. The fluorescence center 1's optimal excitation and emission wavelengths were respectively at $340 \mathrm{~nm}$ and $410 \mathrm{~nm}$ (Figure 2a) with little excitation dependence. However, the fluorescence center 2 was excitation-dependent, whose excitation wavelength started from $380 \mathrm{~nm}$ and emission wavelength concentrated from $445 \mathrm{~nm}$ to the longer. One obvious absorption peak occurred at $326 \mathrm{~nm}$ in the UV-vis absorption spectrum (Figure 2b), which could be attributed to the $\mathrm{n}-\pi^{*}$ transitions of $-\mathrm{C}=\mathrm{O}$. The average fluorescence lifetimes were $6.13 \mathrm{~ns}$ $\left(\mathrm{em}_{1}=410 \mathrm{~nm}\right)$ and $5.05 \mathrm{~ns}\left(\mathrm{em}_{2}=450 \mathrm{~nm}\right)$, indicating they were two different fluorescence centers (Table S3).

Transmission electron microscopy (TEM) images showed that the PCDs had a wide size range from $20 \mathrm{~nm}$ to $30 \mathrm{~nm}$ in diameter (Figure $2 \mathrm{c}$ ). If the sample was too concentrated, only membrane-like structure could be observed, indicating that the polymer feature was reserved. The overwhelming majority of particles were amorphous. Only a few showed lattice fringes with the spacing about $0.19 \pm 0.01 \mathrm{~nm}$ (Figure S1), possibly caused by further slightly carbonization or the crystallization of polymer. From ${ }^{1} \mathrm{H}$ NMR (Figure S2) and ${ }^{13} \mathrm{C}$ NMR spectrum (Figure S3), no characteristic peaks of aromatic structure appeared, which demonstrated that the fluorescence was not from extended conjugation structure but some special groups, namely sub-fluorophores. Owing to such a low carbon-lattice-structure content, no obvious D or G bands could be detected on the Raman spectrum. By analyzing FT-IR spectrum (Figure 2d), the formation of amide bond was confirmed and the functional groups were distinguished as follows, the stretching vibrations of $\mathrm{C}=\mathrm{O}$ at $1643 \mathrm{~cm}^{-1}$, the bending vibrations of $\mathrm{N}-\mathrm{H}$ at $1564 \mathrm{~cm}^{-1}, \mathrm{C}-\mathrm{N}$ at $1391 \mathrm{~cm}^{-1}$ and $\mathrm{N}=\mathrm{O}$ at $1471 \mathrm{~cm}^{-1}$. The X-ray photoelectron spectra of the PCDs showed three peaks at 285.0, 400.1 and $530.6 \mathrm{eV}$, which were attributed to $\mathrm{C} 1 \mathrm{~s}, \mathrm{~N} 1 \mathrm{~s}$ and $\mathrm{O} 1 \mathrm{~s}$, respectively (Figure S4a). The $\mathrm{C} 1 \mathrm{~s}$ spectra (Figure S4b) could be deconvoluted into three peaks, which were attributed to $\mathrm{C}=\mathrm{C} / \mathrm{C}-\mathrm{C}$ bonds $(284.5 \mathrm{eV}), \mathrm{C}-\mathrm{N} / \mathrm{C}-\mathrm{O}$ bonds $(285.6 \mathrm{eV})$ and $\mathrm{C}=\mathrm{O}$ bonds $(287.7 \mathrm{eV})$. The $\mathrm{N} 1 \mathrm{~s}$ spectra (Figure S4c) exhibited three peaks at 399.7, 401.5 and $406.8 \mathrm{eV}$, which were attributed to $\mathrm{C}-\mathrm{N}, \mathrm{N}-\mathrm{H}$ and $\mathrm{N}=\mathrm{O}$ bonds, respectively. The $\mathrm{O} 1 \mathrm{~s}$ spectra (Figure S4d) could be deconvoluted into two peaks, which were attributed to $\mathrm{C}-\mathrm{O}$ bonds $(532.6 \mathrm{eV})$ and $\mathrm{C}=\mathrm{O}$ bonds $(531.1 \mathrm{eV})$. The results were in accordance with FT-IR spectrum analysis. Besides, the thermogravimetric analysis (TGA) was provided (Figure S5). From room temperature to $177^{\circ} \mathrm{C}$, the decrease of total weight might be caused by volatilization of water and desorption of superficial amine. From $177^{\circ} \mathrm{C}$ to $328^{\circ} \mathrm{C}$, the decrease might be caused by the decomposing of amide groups. From $328^{\circ} \mathrm{C}$ to $450^{\circ} \mathrm{C}$, the sharp decrease might result from the pyrolysis of polymer structure. The elemental analysis revealed the average composition is $\mathrm{C} 40.35 \mathrm{wt} \%, \mathrm{H} 7.42 \mathrm{wt} \%, \mathrm{~N} 12.32 \mathrm{wt} \%$ and $\mathrm{O}$ (calculated) $39.91 \mathrm{wt} \%$. All of these observations indicated PCDs was crosslinked polymer nanoparticle with low carbonization degree and high oxygen content. In addition, we also studied cytotoxicity experiment of PCDs (Figure S6). The result showed it had low cell damage (below 500 $\mathrm{mg} / \mathrm{mL}$ ) and could be applied in biological fields.

\subsection{The origin of PCDs' fluorescence}

In order to search for the fluorescence origin of PCDs, we treated PAA and EDA separately at $200^{\circ} \mathrm{C}$ and investigated their products. The PAA hydrothermal product showed weak emission (Figure S7).[36] In contrast, the fluorescence of EDA hydrothermal product (named EDA-H) was much stronger (about 30 times stronger than PAAs'). The PL spectra (Figure 3a) of EDA-H showed two 
fluorescence centers. The fluorescence center 1 was excitation independent and relatively strong, which had optimal excitation and emission wavelengths at $320 \mathrm{~nm}$ and $380 \mathrm{~nm}$. The fluorescence center 2 was excitation dependent and relatively weak, whose excitation wavelength started from $360 \mathrm{~nm}$ and emission wavelength concentrated from $445 \mathrm{~nm}$ to the longer. The average fluorescence lifetimes were $3.69 \mathrm{~ns}\left(\mathrm{em}_{1}=380 \mathrm{~nm}\right)$ and $5.01 \mathrm{~ns}\left(\mathrm{em}_{2}=450 \mathrm{~nm}\right)$, indicating the existence of these two fluorescence centers (Table S3). Besides, the UV-vis absorption spectra (Figure 3b) showed strong peaks at $265 \mathrm{~nm}$ and $321 \mathrm{~nm}$, which could be attributed to the $\mathrm{n}-\pi^{*}$ transitions of $-\mathrm{N}=\mathrm{O}$ and $-\mathrm{C}=\mathrm{O}$, respectively. The similarity of PCDs and EDA-H on optical properties indicated that EDA-H like fluorophores might be the main emission origin of PCDs.

Thus, we emphatically studied the fluorescence of EDA-H. Dialysis was carried out for the purification of EDA-H at first. During dialysis, we found the fluorescent substance could get out of the dialysis bag of $500 \mathrm{D}$, indicating EDA-H was not nanoparticle but fluorescent molecule. Then, we added $\mathrm{K}_{2} \mathrm{~S}_{2} \mathrm{O}_{8}$, as oxidizing agent, to hydrothermal system and found the fluorescence of EDA-H was enhanced. Therefore, it was convinced that more fluorophores were synthesized in hydrothermal process, which might be related to oxidation of amines [37,38]. Then the fluorescence centers of EDA-H were further investigated by adding different amounts of oxidizing agent before hydrothermal reaction and changing $\mathrm{pH}$ value of EDA-H.

The ratio of $-\mathrm{N}=\mathrm{O}$ to $-\mathrm{C}=\mathrm{O}$ increased in the $\mathrm{UV}$-vis absorption spectra with the addition of $\mathrm{K}_{2} \mathrm{~S}_{2} \mathrm{O}_{8}$ (Figure 3b). Simultaneously, the stretching vibration of $\mathrm{N}=\mathrm{O}$ at $1506 \mathrm{~cm}^{-1}$ was getting stronger other than the stretching vibration of $\mathrm{C}=\mathrm{O}$ at $1647 \mathrm{~cm}^{-1}$ and the bending vibration of $\mathrm{N}-\mathrm{H}$ at $1588 \mathrm{~cm}^{-1}$ in the FT-IR analysis (Figure 3c). It meaned that more $\mathrm{N}=\mathrm{O}$ generated during more drastic oxidation process. For fluorescence, the change of two centers' relative proportion and red-shifted emission could be observed intuitively in excitation-emission matrix (Figure 4). Based on the discussion above, we assumed that center 1 might originate from $-\mathrm{C}=\mathrm{O}$ related fluorophores (mainly amide and/or its derived structures) and center 2 might be resulted from $-\mathrm{N}=\mathrm{O}$ related fluorophores. To verify our conjecture further, different amounts of hydrochloric acid were added into EDA-H to tune the solution from basic to acid. Subsequently, we recorded the intensity of the two fluorescence centers (Figure 3d) and found that center 1 was affected greatly other than center 2 . This phenomenon might be caused by protonation and deprotonation of amide, which was in good agreement with the previous analysis.

\subsection{The enhanced emission of PCDs by polymer structure}

Based on the discussions above, the strong emission peak of PCDs at $410 \mathrm{~nm}$ was assumed to be similar to the center 1 of EDA-H $(-\mathrm{C}=\mathrm{O}$ related fluorophores, mainly amide and/or its derived structures). However, compared with EDA-H, it was obvious that PCDs had increased quantum yield (from $25.29 \%$ to $44.18 \%$ ) and red-shifted emission (from $380 \mathrm{~nm}$ to $410 \mathrm{~nm}$ ). We considered the PCDs' fluorescence might be caused by the synergistic effect of PAA's polymer chains and EDA-H's fluorescence centers. Thus, some experiments were designed to support our conjectures as follows.

Firstly, the temperature dependent PL spectra of EDA-H was investigated (Figure S8). The result showed that the fluorescence intensity distinctly decreased with the increase of temperature, indicating that the activation of fluorophores' vibration and rotation was a dominant factor in fluorescence intensity. Thus, it was reasonable to consider that the polymer chains (especially crosslinked polymer structure) could also immobilize fluorophores and restrain vibration and rotation, eventually leading to the enhanced emission. This effect was named CEE effect in our previous reports [33]. Then, we further calculated EDA-H and PCDs' rate constants of radiative transition and non-radiation transition according to their fluorescence lifetimes and absolute quantum yields (Table S3). The radiative transition rate constants of EDA-H and PCDs were calculated as $0.0685 \mathrm{~ns}^{-1}$ and $0.0721 \mathrm{~ns}^{-1}$, suggesting they owned similar emission centers. The non-radiative transition rate constants of EDA-H and PCDs were $0.2025 \mathrm{~ns}^{-1}$ and $0.0912 \mathrm{~ns}^{-1}$, suggesting the process of PCDs' non-radiation transition was obviously inhibited.

Next, PCDs was dissolved in different mixed liquids of ethanol and water in order to prove the existence of external polymer chains. With the increase of ethanol's proportion, the turbidity appeared and the emission intensity was enhanced (Figure 5). We inferred that the polymer chains were shrunk in poor solvent, which immobilized fluorophores and enhanced the emission. Furthermore, as the increase of ethanol's proportion, the solubility of hydrophilic PCDs decreased and PCDs began to separate out. The aggregation of PCDs could further enhance the emission. These explanations were supported by DLS analysis (Table S4).

Furthermore, to illustrate the function of polymer structure, we investigated the PL emission by 
dissolving EDA-H in different solvents (Table 1). The emission peak was found to be red-shifted and broadened in some solvents, which might result from the dipolar interaction of the solvent molecule and fluorophores [39]. However, the solvent's influence on emission peak of PCDs was not obvious (Table 1). The basically unchanged peak position and FWHM of PCDs indicated that the fluorophores were surrounded and protected by polymer chains, so the solvent molecule couldn't approach them. What's more, the polymer chains possessed plenty of carboxyl groups which could also induce the redshift of fluorophores.

In brief, the fluorescence of PCDs mainly derived from $-\mathrm{C}=\mathrm{O}$ related fluorophores (mainly amide and/or its derived structures) like EDA-H'. During the hydrothermal process, PAA formed a crosslinked structure and hold the fluorophores inside. On one hand, the polymer structure immobilized the fluorophores and enhanced the emission. On the other hand, polymer structure provided a new chemical environment and shifted the emission from UV region to visible region. The center 2 of PCDs which was excitation-dependent and relatively weak, might also derive from EDA-H's center 2. The relevant investigation is on the way.

\section{Conclusion}

In summary, we got a new kind of PCDs with high QY about $44.18 \%$ via a one-pot hydrothermal method. The properties of the obtained PCDs were characterized in detail and the fluorescence mechanism of PCDs was studied. In comparison with EDA-H, the PCDs was confirmed to own similar fluorescence centers. By crosslinking and wrapping the fluorescence centers, PAA's chains could reduce vibration and rotation of fluorephores and provide with a stable chemical environment. Thus, the improved quantum yield and red-shifted emission were attributed to the effect of polymer chains. Generally speaking, the crosslinked polymer structure could not only enhance the emission [33] but also redshift the emission peaks, which further enriched the explanation of CEE effect. Fluorescence mechanism of PCDs is a mysterious but interesting topic, thus more research works are highly desired.

\section{Acknowledgements}

This work was financially supported by the National key research and development program of China (2016YFB0401701) and the National Science Foundation of China (NSFC) under Grant Nos. 51433003, 51373065, 21504029.

\section{Keywords}

polymer carbon dots; fluorescence; hydrothermal; mechanism; crosslink enhanced emission.

\section{References}

[1] S. N. Baker, G. A. Baker, Luminescent carbon nanodots: emergent nanolights. Angew Chem Int Ed Engl 49 (2010) 6726-6744.

[2] H. Li, Z. Kang, Y. Liu, S.-T. Lee, Carbon nanodots: synthesis, properties and applications. J Mater Chem 22 (2012) 24230.

[3] X. T. Zheng, A. Ananthanarayanan, K. Q. Luo, P. Chen, Glowing Graphene Quantum Dots and Carbon Dots: Properties, Syntheses, and Biological Applications. Small 14 (2014) 1620-1636.

[4] S. Y. Lim, W. Shen, Z. Gao, Carbon quantum dots and their applications. Chem Soc Rev 44 (2015) 362-381.

[5] A. Zhao, Z. W. Chen, X. G. Qu, Recent advances in bioapplications of C-dots. Carbon 85 (2015) 309-327.

[6] P. Miao, K. Han, W. B. Cheng, Recent advances in carbon nanodots: synthesis, properties and biomedical applications. Nanoscale 7 (2015) 1586-1595.

[7] Y. Yang, J. H. Cui, Y. L. Liu, One-step synthesis of amino-functionalized fluorescent carbon nanoparticles by hydrothermal carbonization of chitosan. Chem Commun (Camb) 48 (2012) 380-382. 
[8] W. Li, Z. H. Zhang, D. Y. Zhao, Simple and green synthesis of nitrogen-doped photoluminescent carbonaceous nanospheres for bioimaging. Angew Chem Int Ed Engl 52 (2013) 8151-8155.

[9] A.-M. Alam, Y. Liu, M. Park, S.-J. Park, H.-Y. Kim, Preparation and characterization of optically transparent and photoluminescent electrospun nanofiber composed of carbon quantum dots and polyacrylonitrile blend with polyacrylic acid. Polymer 59 (2015) 35-41.

[10] D. Li, D. Han, D. Z. Shen, Supra-(carbon nanodots) with a strong visible to near-infrared absorption band and efficient photothermal conversion. Light: Science \& Applications 5 (2016) e16120.

[11] X. Xu, R. Ray, W. A. Scrivens, Electrophoretic analysis and purification of fluorescent single-walled carbon nanotube fragments. J Am Chem Soc 126 (2004) 12736-12737.

[12] S. J. Zhu, Q. N. Meng, B. Yang, Highly photoluminescent carbon dots for multicolor patterning, sensors, and bioimaging. Angew Chem Int Ed Engl 52 (2013) 3953-3957.

[13] L. L. Pan, S. Sun, A. D. Zhang, H. W. Lin, Truly Fluorescent Excitation-Dependent Carbon Dots and Their Applications in Multicolor Cellular Imaging and Multidimensional Sensing. Adv Mater 27 (2015) 7782-7787.

[14] J. Ge, Q. Y. Jia, P. F. Wang, Red-Emissive Carbon Dots for Fluorescent, Photoacoustic, and Thermal Theranostics in Living Mice. Adv Mater 27 (2015) 4169-4177.

[15] S. N. Qu, D. Zhou, W. Y. Ji, D. Z. Shen, Toward Efficient Orange Emissive Carbon Nanodots through Conjugated sp(2) -Domain Controlling and Surface Charges Engineering. Adv Mater 28 (2016) 3516-3521.

[16] K. Jiang, S. Sun, L. Zhang, H. W. Lin, Red, green, and blue luminescence by carbon dots: full-color emission tuning and multicolor cellular imaging. Angew Chem Int Ed Engl 54 (2015) 5360-5363.

[17] S. J. Zhu, Y. B. Song, X. H. Zhao, B. Yang, The photoluminescence mechanism in carbon dots (graphene quantum dots, carbon nanodots, and polymer dots): current state and future perspective. Nano Research 8 (2015) 355-381.

[18] L. Bao, C. Liu, Z. L. Zhang, D. W. Pang, Photoluminescence-tunable carbon nanodots: surface-state energy-gap tuning. Adv Mater 27 (2015) 1663-1667.

[19] Y. B. Song, S. J. Zhu, B. Yang, Investigation from chemical structure to photoluminescent mechanism: a type of carbon dots from the pyrolysis of citric acid and an amine. J. Mater. Chem. C 3 (2015) 5976-5984.

[20] H. Kim, Y. Kim, J. Y. Chang, Polymers for Luminescent Sensing Applications. Macromolecular Chemistry And Physics 215 (2014) 1274-1285.

[21] H. N. Kim, Z. Guo, W. Zhu, J. Yoon, H. Tian, Recent progress on polymer-based fluorescent and colorimetric chemosensors. Chem Soc Rev 40 (2011) 79-93.

[22] F. Zheng, M. Wen, F. Zeng, S. Wu, A water-soluble, low-cytotoxic and sensitive fluorescent probe based on poly(ethylene glycol) for detecting sulfide anion in aqueous media and imaging inside live cells. Polymer 54 (2013) 5691-5697.

[23] J. H. Son, G. Jang, T. S. Lee, Synthesis of water-soluble, fluorescent, conjugated polybenzodiazaborole for detection of cyanide anion in water. Polymer 54 (2013) 3542-3547.

[24] C. Wu, S. J. Hansen, D. T. Chiu, Design of highly emissive polymer dot bioconjugates for in vivo tumor targeting. Angew Chem Int Ed Engl 50 (2011) 3430-3434.

[25] I. Grabchev, V. Bojinov, J.-M. Chovelon, Synthesis, photophysical and photochemical properties of fluorescent poly(amidoamine) dendrimers. Polymer 44 (2003) 4421-4428.

[26] B.-B. Wang, X.Zhang, Y. Wei, Poly(amidoamine) dendrimers with phenyl shells: fluorescence and aggregation behavior. Polymer 45 (2004) 8395-8402.

[27] W. Yang, C. Y. Pan, M. D. Luo, H. B. Zhang, Fluorescent mannose-functionalized hyperbranched poly(amido amine)s: synthesis and interaction with E. coli. Biomacromolecules 11 (2010) 1840-1846.

[28] W. Yang, C. Y. Pan, Synthesis and Fluorescent Properties of Biodegradable Hyperbranched Poly(amido 
amine)s. Macromolecular rapid communications 30 (2009) 2096-2101.

[29] R. B. Wang, W. Z. Yuan, X. Y. Zhu, Aggregation-induced emission of non-conjugated poly(amido amine)s: Discovering, luminescent mechanism understanding and bioapplication. Chinese Journal of Polymer Science 33 (2015) 680-687.

[30] D. Wang, T. Imae, Fluorescence emission from dendrimers and its pH dependence. J Am Chem Soc 126 (2004) 13204-13205.

[31] G. Song, Y. N. Lin, H. L.Wang, Strong fluorescence of poly(N-vinylpyrrolidone) and its oxidized hydrolyzate. Macromolecular rapid communications 36 (2015) 278-285.

[32] E. Zhao, Jacky W. Y. Lam, B. Z. Tang, Poly[(maleic anhydride)-alt-(vinyl acetate)]: A Pure Oxygenic Nonconjugated Macromolecule with Strong Light Emission and Solvatochromic Effect. Macromolecules 48 (2015) 64-71.

[33] S. J. Zhu, Y. B. Song, J. R. Shao, B. Yang, Non-Conjugated Polymer Dots with Crosslink-Enhanced Emission in the Absence of Fluorophore Units. Angew Chem Int Ed Engl 54 (2015) 14626-14637.

[34] S. J. Zhu, L. Wang, N. Zhou, B. Yang, The crosslink enhanced emission (CEE) in non-conjugated polymer dots: from the photoluminescence mechanism to the cellular uptake mechanism and internalization. Chem Commun 50 (2014) 13845-13848.

[35] Y. B. Song, S. J. Zhu, J. R. Shao, B. Yang, Polymer carbon dots-a highlight reviewing their unique structure, bright emission and probable photoluminescence mechanism Polymer Science Part A: Polymer Chemistry (2016) DOI: 10.1002/pola.20160550.

[36] S. J. Zhu, J. H. Zhang, L. Wang, B. Yang, A general route to make non-conjugated linear polymers luminescent. Chem Commun (Camb) 48 (2012) 10889-10891.

[37] G. S. Song, Y. N. Lin, H. L. Wang, Strong fluorescence of poly(N-vinylpyrrolidone) and its oxidized hydrolyzate. Macromolecular rapid communications 36 (2015) 278-285.

[38 M. Sun, C. Y. Hong, C. Y. Pan, A unique aliphatic tertiary amine chromophore: fluorescence, polymer structure, and application in cell imaging. J Am Chem Soc 134 (2012) 20581-20584.

[39] D. Y. Pan, J. C. Zhang, Z. Li, M. H. Hong, Observation of pH-, solvent-, spin-, and excitation-dependent blue photoluminescence from carbon nanoparticles. Chemical Communications 46 (2010) 3681. 


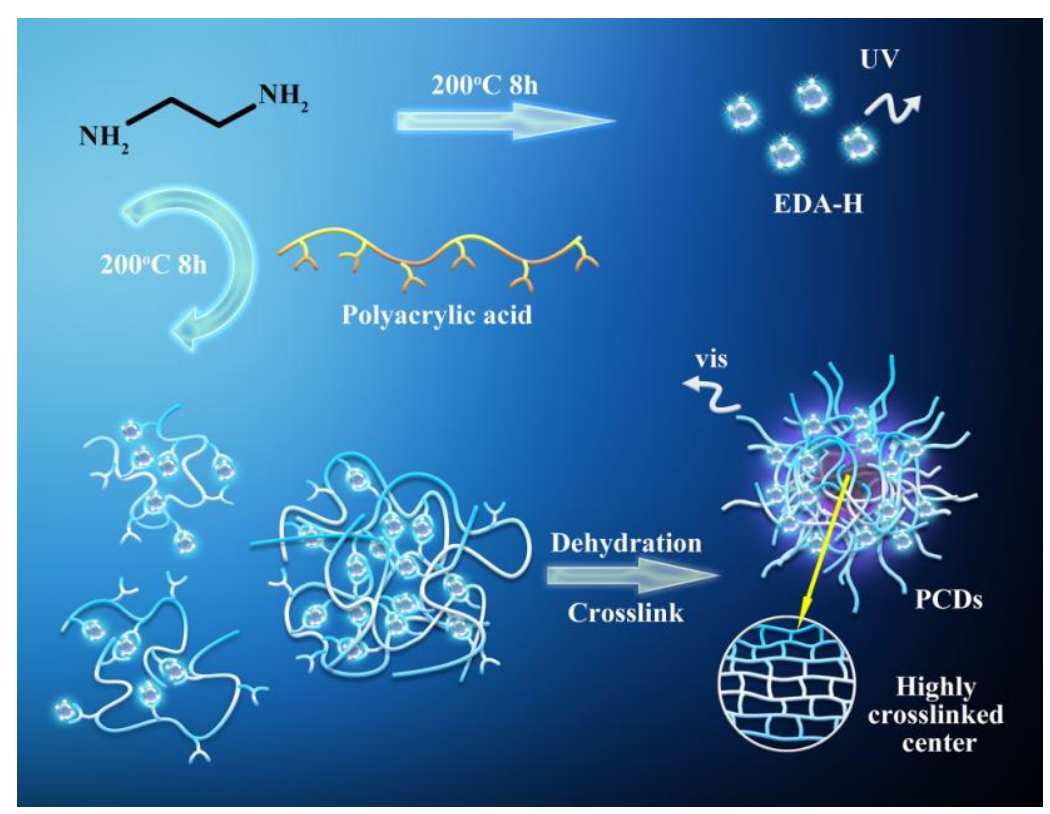

Figure 1 The schematic diagram of experimental procedure.

(a)

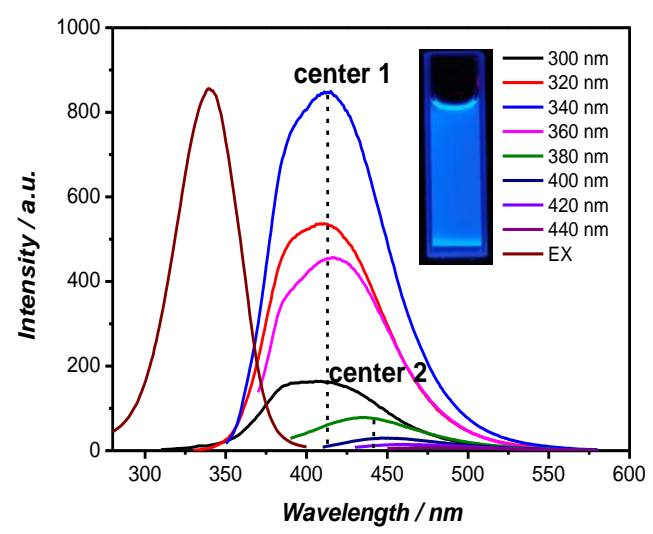

(c)

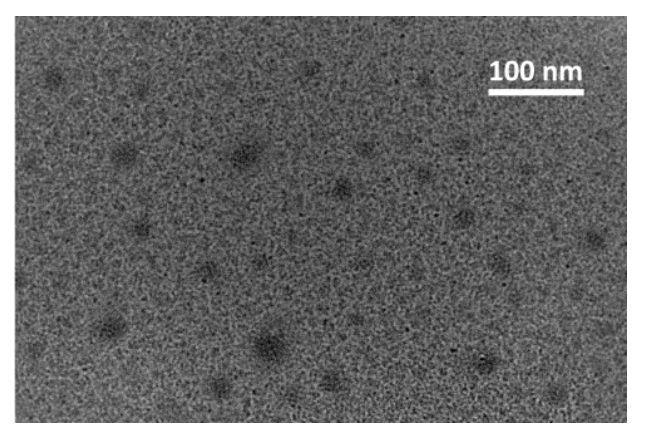

(b)

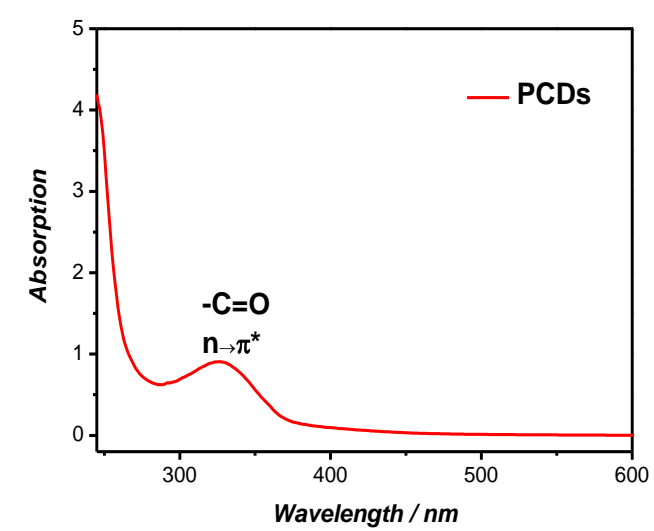

(d)

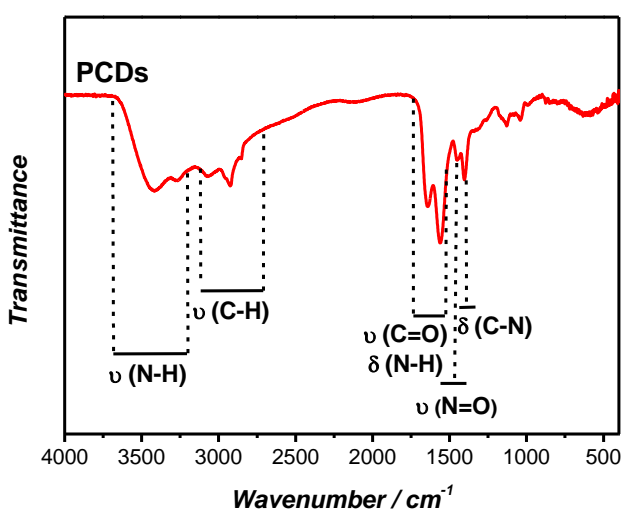

Figure 2 The characterizations of PCDs include a) PL excitation (EX) and emission (300-440 nm) spectra, b) UV-vis absorption spectrum, c) high-resolution TEM image and d) FT-IR spectrum. 
(a)

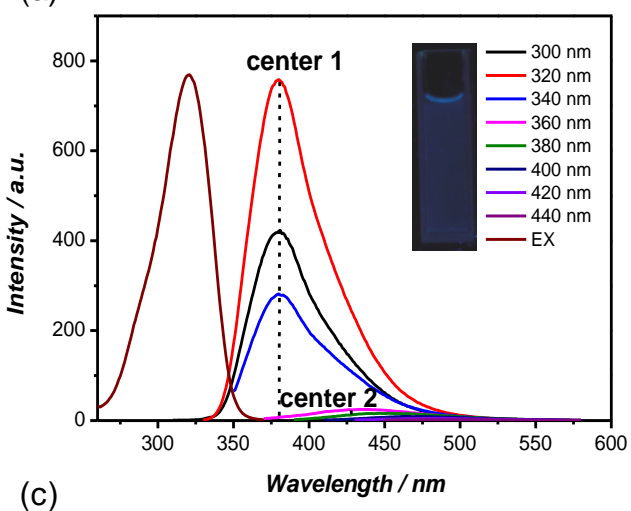

(c)

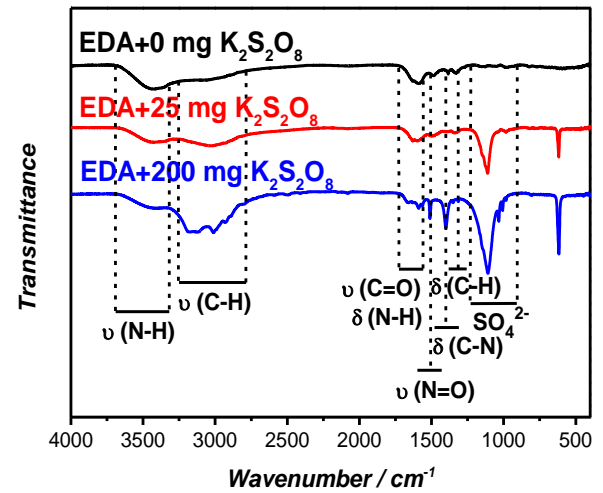

(b)

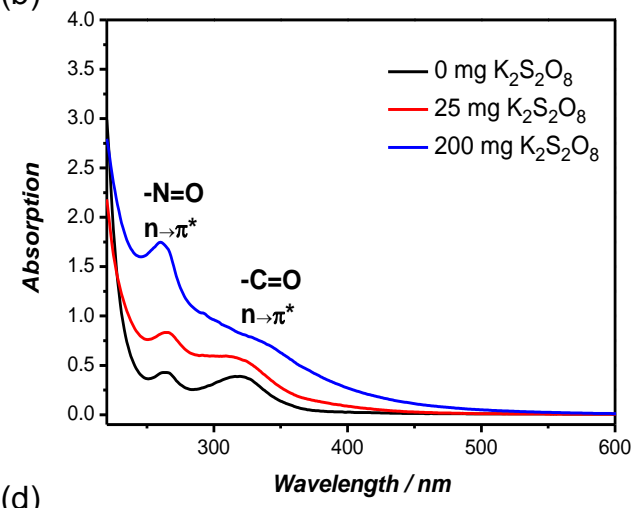

(d)

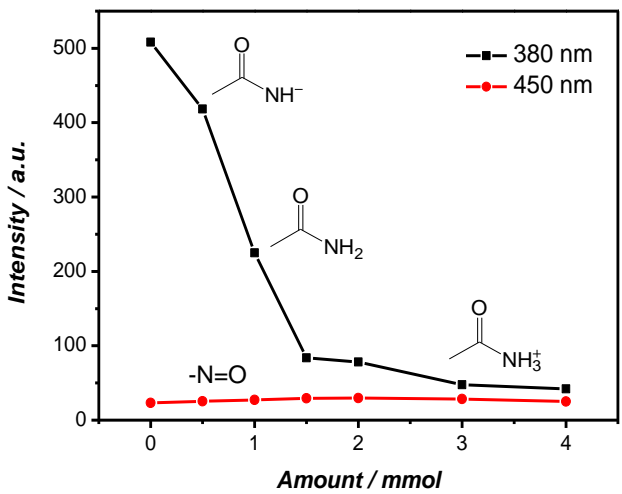

Figure 3 a) The PL excitation (EX) and emission (300-440 nm) spectra of the EDA-H. b) The UV-vis absorption spectra of the EDA hydrothermal products with different amounts of $\mathrm{K}_{2} \mathrm{~S}_{2} \mathrm{O}_{8}$. c) The FT-IR spectra of the EDA hydrothermal products with different amounts of $\mathrm{K}_{2} \mathrm{~S}_{2} \mathrm{O}_{8}$. d) The PL intensity of EDA-H's two emission peaks $\left(\mathrm{em}_{1}=380 \mathrm{~nm}, \mathrm{em}_{2}=450 \mathrm{~nm}\right)$ with different amounts of hydrogen ion. 
(a)

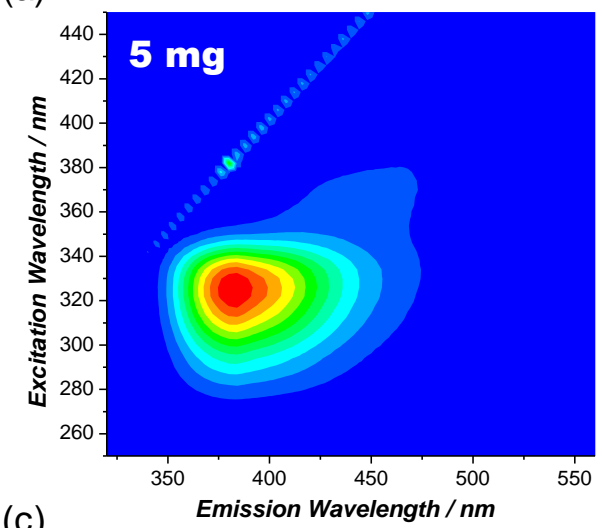

(c)
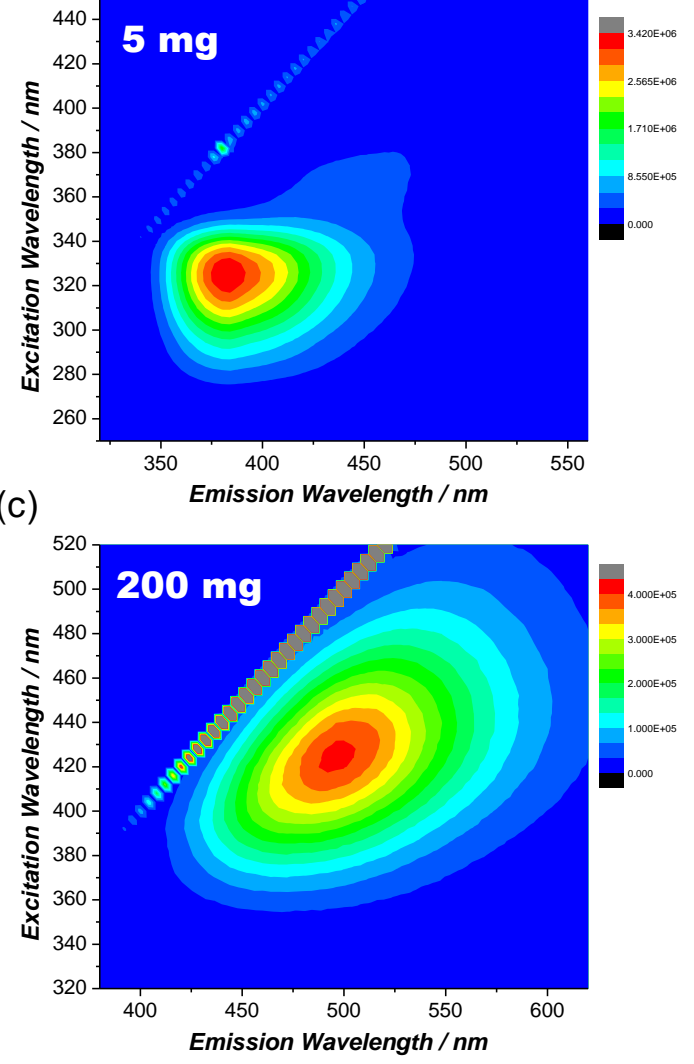

(b)

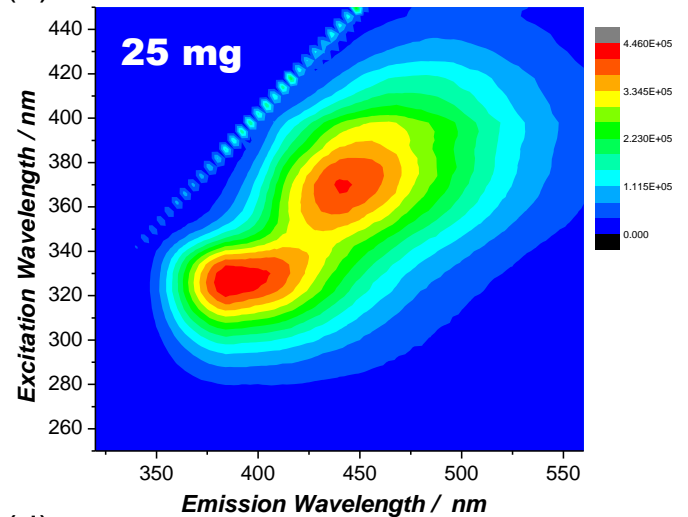

(d)

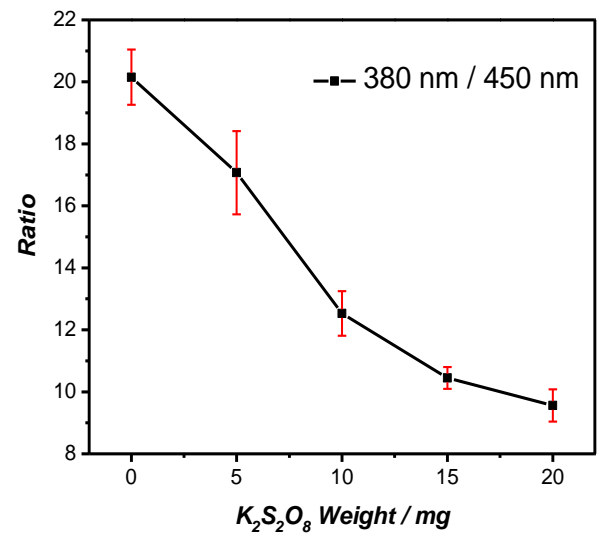

Figure 4 Excitation-emission matrix for the EDA hydrothermal products with different amounts of $\mathrm{K}_{2} \mathrm{~S}_{2} \mathrm{O}_{8}$, a) $5 \mathrm{mg}$, b) $25 \mathrm{mg}$, c) $200 \mathrm{mg}$ and d) The ratio of two centers' PL intensity $\left(\mathrm{em}_{1}=380 \mathrm{~nm}, \mathrm{em}_{2}=450 \mathrm{~nm}\right)$.

(a)

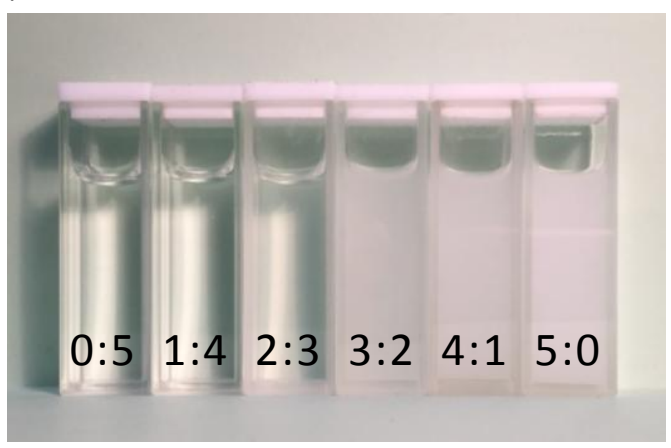

(b)

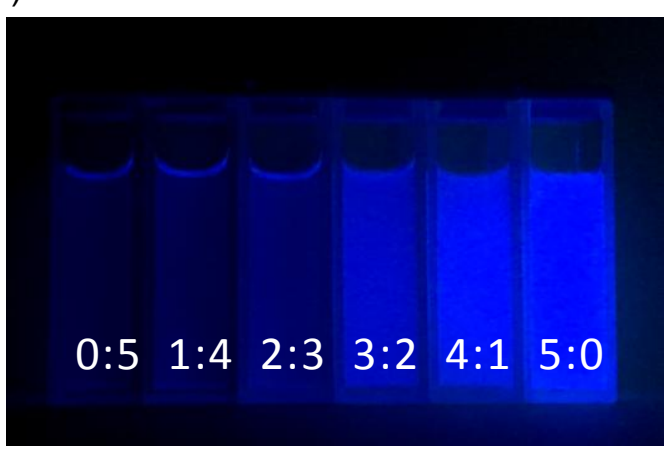

Figure 5 The images of the PCDs dissolved in different ratios of ethanol to DI water a) under natural light and b) under handheld UV lamp (ex=365 nm). 


\begin{tabular}{cccccccc}
\hline & solvent & ethyl acetate & tetrahydrofuran & isopropanol & acetone & ethonal & water \\
\hline EDA-H & peak $(\mathrm{nm})$ & 382.84 & 390.30 & 384.40 & 393.88 & 383.41 & 380.83 \\
& FWHM $(\mathrm{nm})$ & 61.16 & 74.23 & 60.34 & 84.53 & 57.89 & 54.60 \\
\multirow{2}{*}{ PCDs } & peak (nm) & 410.57 & 410.23 & 409.67 & 411.18 & 409.64 & 410.36 \\
& FWHM (nm) & 80.32 & 79.27 & 81.90 & 84.80 & 81.82 & 80.28 \\
\hline
\end{tabular}

Table 1 The statistical table of PL intensity and FWHM in different solvents. 
A New Type of Polymer Carbon Dots with High Quantum Yield: from Synthesis to Investigation on Fluorescence Mechanism

Songyuan Tao, Yubin Song, Shoujun Zhu, Jieren Shao, Bai Yang*

In this article, a new type of polymer carbon dots was synthesized with quantum yield as high as $44.18 \%$. The properties were characterized in detail and probable fluorescence source was inferred. Moreover, the function of polymer chains towards the improved quantum yield and red-shifted emission was discussed, which can provide with a new understanding on crosslink enhanced emission (CEE) effect in non-conjugated system.

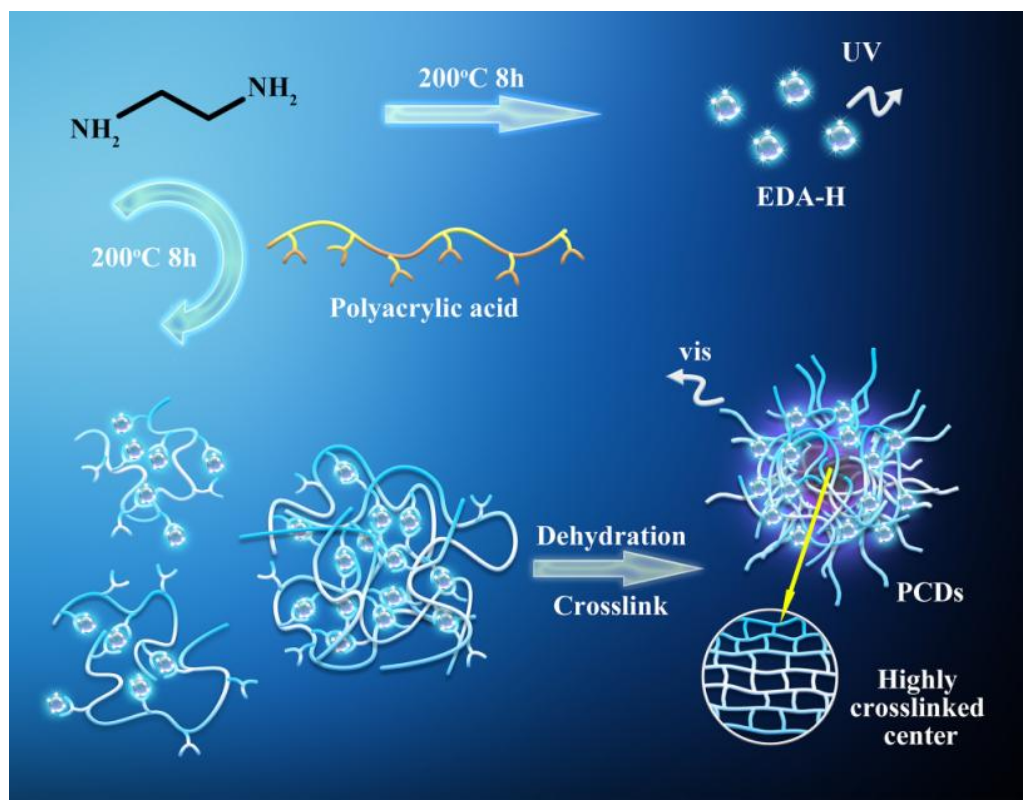

